\title{
ON THE SUMS OF COMPOUND NEGATIVE BINOMIAL AND GAMMA RANDOM VARIABLES
}

\author{
P. VELLAISAMY ${ }^{* * *}$ AND \\ N. S. UPADHYE, ${ }^{*}$ Indian Institute of Technology Bombay
}

\begin{abstract}
We study the convolution of compound negative binomial distributions with arbitrary parameters. The exact expression and also a random parameter representation are obtained. These results generalize some recent results in the literature. An application of these results to insurance mathematics is discussed. The sums of certain dependent compound Poisson variables are also studied. Using the connection between negative binomial and gamma distributions, we obtain a simple random parameter representation for the convolution of independent and weighted gamma variables with arbitrary parameters. Applications to the reliability of $m$-out-of- $n$ :G systems and to the shortest path problem in graph theory are also discussed.
\end{abstract}

Keywords: Compound negative binomial distribution; convolution; random parameter representation; compound Poisson distribution; sums of gamma random variables; Poisson process

2000 Mathematics Subject Classification: Primary 60E05

Secondary 62E15

\section{Introduction}

The compound negative binomial (CNB) model arises naturally in several fields, such as insurance mathematics and actuarial sciences, and has been studied by several authors. For a recent reference, see Drekic and Willmot (2005) and the references therein. It also arises in nonactuarial applications (see Johnson et al. (2005, pp. 232-250) and Vellaisamy and Upadhye (2007)). Recently, Furman (2007) studied the sums of independent negative binomial random variables and obtained an interesting recurrence relation for computing its probability mass function (PMF). He also showed that the convolution of a negative binomial distribution with arbitrary parameters is a negative binomial distribution, but with a random parameter.

In Section 2 we first derive an exact expression for the distribution of sums of CNB random variables. For the negative binomial (NB) case, this expression reduces to a finite-sum expression which is numerically compared with the series expression of Furman (2007). We also obtain a simple random parameter representation for the convolution of CNB distributions, where the compounding distributions $Q_{j}=Q$. Theorems 2.1 and 2.2 of Furman (2007) follow as special cases. Our approach is essentially that of Furman (2007), except that we use the distribution itself rather than using its moment generating function (MGF).

If the $Q_{j}$ s are different then the convolution of CNB distributions is neither a CNB nor a mixture of CNBs. In such cases, a compound Poisson (CP) representation is presented. It is also shown that a sum of certain dependent $\mathrm{CP}$ variables is again a $\mathrm{CP}$ variable. It is well known

Received 18 September 2008; revision received 21 January 2009.

* Postal address: Department of Mathematics, Indian Institute of Technology Bombay, Powai, Mumbai, 400 076, India.

** Email address: pv@math.iitb.ac.in 
that the weighted sums of independent gamma variables arise in several contexts in probability and statistics. The reader may refer to Diaconis and Perlman (1990) for several examples and applications. They mentioned that the distribution of such a sum is not expressible in a closed form and so discussed approximations and studied tail probabilities. Using the connection between the NB and gamma distributions (see Engel and Zijlstra (1980) or Vellaisamy and Sreehari (2008)), we obtain, in Section 3, the exact distribution of weighted sums of independent gamma random variables with arbitrary parameters.

In Section 4 we discuss several interesting examples and applications of the results obtained in Sections 2 and 3. The problems of total claim amount and the distribution of combined portfolios, which arise in insurance mathematics, are discussed as applications of convolutions of $\mathrm{CNB}$ variables and certain dependent $\mathrm{CP}$ variables, respectively. Furthermore, two important applications of gamma convolutions, namely, the reliability of $m$-out-of- $n$ :G systems with dynamic failure rates and the shortest path problem in graph theory, are analyzed in detail. At the end, the main contributions of the paper are briefly outlined.

\section{Convolution of CNB variables}

Let $\mathbb{Z}_{+}=\{0,1, \ldots\}$ be the set of nonnegative integers, let $0<p<1$, and let $q=(1-p)$. Let $N \sim \mathrm{NB}(\alpha, p)$, the $\mathrm{NB}$ distribution with

$$
\mathrm{P}(N=m)=\left(\begin{array}{c}
\alpha+m-1 \\
m
\end{array}\right) p^{\alpha} q^{m}, \quad m \in \mathbb{Z}_{+}, \alpha>0,0<p<1 .
$$

Then, a real-valued random variable $Y$ is said to follow a CNB distribution with parameters $\alpha$, $p$, and $Q$, denoted by $\operatorname{CNB}(\alpha, p, Q)$, if it admits the random sum representation $Y=\sum_{i=1}^{N} W_{i}$, where $N \sim \mathrm{NB}(\alpha, p)$ and $\left\{W_{i}\right\}$ is a sequence of independent and identically distributed (i.i.d.) random variables with distribution $Q$ that is independent of $N$.

Let $m \in \mathbb{Z}_{+}$, let $\delta_{m}$ be the Dirac measure concentrated at $m$, and let $Q^{m}$ denote the $m$-fold convolution of $Q$. Then, the distribution $\mathcal{L}(Y)$ of $Y$ is given by

$$
\begin{aligned}
\mathscr{L}(Y)(A) & =\sum_{m=0}^{\infty}\left(\begin{array}{c}
\alpha+m-1 \\
m
\end{array}\right) p^{\alpha}(q Q)^{m}(A) \\
& =p^{\alpha} \sum_{m=0}^{\infty}(-1)^{m}\left(\begin{array}{c}
-\alpha \\
m
\end{array}\right)(q Q)^{m}(A) \\
& =p^{\alpha}\left(\delta_{0}-q Q\right)^{-\alpha}(A) \\
& =\left(\delta_{0}-\frac{q}{p}\left(Q-\delta_{0}\right)\right)^{-\alpha}(A),
\end{aligned}
$$

where $A$ is any Borel-measurable set. Indeed, (2.3) is a formal representation for (2.2) in the sense that, when (2.3) is expanded as a power series, the powers of $Q$ represent its convolutions.

When $Q=\delta_{1}, Q^{m}=\delta_{1}^{m}=\delta_{m}$ and, hence, from (2.2), $\operatorname{CNB}\left(\alpha, p, \delta_{1}\right)=\mathrm{NB}(\alpha, p)$. Also, when $\alpha=1, \operatorname{CNB}(1, p, Q):=\mathrm{CG}(p, Q)$ denotes the compound geometric distribution with parameters $p$ and $Q$.

First we obtain an exact representation for the convolution of CNB distributions with $Q_{j}=Q$. 
Theorem 2.1. Let $Y_{j} \sim \operatorname{CNB}\left(\alpha_{j}, p_{j}, Q\right)$ for $1 \leq j \leq n$, and let $S_{n}=\sum_{j=1}^{n} Y_{j}$. Then

$$
\mathrm{P}\left(S_{n} \leq x\right)=\sum_{l=0}^{\infty}\left(\sum_{m_{1}+\cdots+m_{n}=l} \prod_{j=1}^{n}\left(\begin{array}{c}
\alpha_{j}+m_{j}-1 \\
m_{j}
\end{array}\right) p_{j}^{\alpha_{j}} q_{j}^{m_{j}}\right) Q^{l}((-\infty, x]),
$$

where the inside sum is over nonnegative integers $m_{j}$ such that $m_{1}+m_{2}+\cdots+m_{n}=l$.

Proof. The proof is by induction. Note that, for $n=1,(2.4)$ reduces to the distribution function of $Y_{1}$. Assume that (2.4) is true for $n=k-1$. Then

$$
\begin{aligned}
& \mathrm{P}\left(S_{k} \leq x\right)=\int_{\mathbb{R}} \mathrm{P}\left(S_{k-1} \leq x-y\right) \mathrm{d} F_{Y_{k}}(y) \\
& =\int_{\mathbb{R}} \sum_{l=0}^{\infty}\left(\sum_{m_{1}+\cdots+m_{k-1}=l} \prod_{j=1}^{k-1}\left(\begin{array}{c}
\alpha_{j}+m_{j}-1 \\
m_{j}
\end{array}\right) p_{j}^{\alpha_{j}} q_{j}^{m_{j}}\right) Q^{l}((-\infty, x-y]) \\
& \times \sum_{m_{k}=0}^{\infty}\left(\begin{array}{c}
\alpha_{k}+m_{k}-1 \\
m_{k}
\end{array}\right) p_{k}^{\alpha_{k}} q_{k}^{m_{k}} \mathrm{~d} Q^{m_{k}}(y) \\
& =\sum_{l=0}^{\infty} \sum_{m_{k}=0}^{\infty}\left(\sum_{m_{1}+\cdots+m_{k-1}=l} \prod_{j=1}^{k-1}\left(\begin{array}{c}
\alpha_{j}+m_{j}-1 \\
m_{j}
\end{array}\right) p_{j}^{\alpha_{j}} q_{j}^{m_{j}}\right)\left(\begin{array}{c}
\alpha_{k}+m_{k}-1 \\
m_{k}
\end{array}\right) \\
& \times p_{k}^{\alpha_{k}} q_{k}^{m_{k}} \int_{\mathbb{R}} Q^{l}((-\infty, x-y]) \mathrm{d} Q^{m_{k}}(y) \\
& =\sum_{l=0}^{\infty} \sum_{m_{k}=0}^{\infty}\left(\sum_{m_{1}+\cdots+m_{k-1}=l} \prod_{j=1}^{k-1}\left(\begin{array}{c}
\alpha_{j}+m_{j}-1 \\
m_{j}
\end{array}\right) p_{j}^{\alpha_{j}} q_{j}^{m_{j}}\right)\left(\begin{array}{c}
\alpha_{k}+m_{k}-1 \\
m_{k}
\end{array}\right) \\
& \times p_{k}^{\alpha_{k}} q_{k}^{m_{k}} Q^{l+m_{k}}((-\infty, x]) \\
& =\sum_{r=0}^{\infty}\left(\sum_{m_{1}+\cdots+m_{k}=r} \prod_{j=1}^{k}\left(\begin{array}{c}
\alpha_{j}+m_{j}-1 \\
m_{j}
\end{array}\right) p_{j}^{\alpha_{j}} q_{j}^{m_{j}}\right) Q^{r}((-\infty, x]),
\end{aligned}
$$

where the last equality follows by substituting $l+m_{k}=r$ and then interchanging the order of summation of $m_{k}$ and $r$. Thus, (2.4) is satisfied for $n=k$, which completes the proof.

Remark 2.1. When $Q=\delta_{1},(2.4)$ reduces to

$$
\mathrm{P}\left(S_{n} \leq x\right)=\sum_{l=0}^{\lfloor x\rfloor}\left(\sum_{m_{1}+\cdots+m_{n}=l} \prod_{j=1}^{n}\left(\begin{array}{c}
\alpha_{j}+m_{j}-1 \\
m_{j}
\end{array}\right) p_{j}^{\alpha_{j}} q_{j}^{m_{j}}\right),
$$

where $\lfloor x\rfloor$ denotes the integral part of $x$.

Also, the PMF of $S_{n}$ is

$$
\mathrm{P}\left(S_{n}=x\right)=\sum_{m_{1}+\cdots+m_{n}=x} \prod_{j=1}^{n}\left(\begin{array}{c}
\alpha_{j}+m_{j}-1 \\
m_{j}
\end{array}\right) p_{j}^{\alpha_{j}} q_{j}^{m_{j}} \text { for } x \in \mathbb{Z}_{+} .
$$

An alternative form for $\mathrm{P}\left(S_{n}=x\right)$ is given in Furman (2007, Equation (11)), which is a series whose coefficients are recursively defined. In contrast, our expression (2.5) is compact and the exact value can be easily computed. 
TABLE 1: The computation of $p_{\mathrm{VU}}=\mathrm{P}\left(S_{n}=x\right)$ using (2.5).

\begin{tabular}{|c|c|c|c|c|c|c|c|c|c|c|}
\hline \multirow{2}{*}{$n$} & \multicolumn{2}{|c|}{$x=3$} & \multicolumn{2}{|c|}{$x=5$} & \multicolumn{2}{|c|}{$x=8$} & \multicolumn{2}{|c|}{$x=10$} & \multicolumn{2}{|c|}{$x=15$} \\
\hline & $p_{\mathrm{VU}}$ & Time & $p_{\mathrm{VU}}$ & Time & $p_{\mathrm{VU}}$ & Time & $p_{\mathrm{VU}}$ & Time & $p_{\mathrm{VU}}$ & Time \\
\hline 2 & 0.0232 & 0. & 34 & 0.00 & 042 & 0.000 & 0.0442 & 0.000 & 0.03856 & 0.000 \\
\hline 3 & 0.00273 & 0. & 0730 & 0.015 & 4 & .000 & 0.02 & 0.0 & 07 & 0.000 \\
\hline 4 & 020 & 0.0 & 4 & 0. & 8 & 0 & 0 & 0 & 99 & 0.015 \\
\hline 5 & 0.00001 & 0.0 & 0010 & 0.016 & 0076 & 0.000 & 0.0 & 0.015 & 0.00 & 0.063 \\
\hline 6 & 0.00000 & 0.0 & 0.00001 & 0.016 & 0.00014 & 0.031 & 0.00047 & 0.047 & 0.00365 & 0.234 \\
\hline 7 & 0.00000 & 0.0 & 0.00000 & 0.016 & 0.00003 & 0.063 & 0.00013 & 0.140 & 0.00154 & 0.844 \\
\hline
\end{tabular}

TABLE 2: The computation of $p_{\mathrm{F}}=\mathrm{P}\left(S_{n}=x\right)$ using Furman's formula (11).

\begin{tabular}{|c|c|c|c|c|c|c|c|c|c|c|}
\hline \multirow{2}{*}{$n$} & \multicolumn{2}{|c|}{$x=3$} & \multicolumn{2}{|c|}{$x=5$} & \multicolumn{2}{|c|}{$x=8$} & \multicolumn{2}{|c|}{$x=10$} & \multicolumn{2}{|c|}{$x=15$} \\
\hline & $p_{\mathrm{F}}$ & Time & $p_{\mathrm{F}}$ & Time & $p_{\mathrm{F}}$ & Time & $p_{\mathrm{F}}$ & Time & $p_{\mathrm{F}}$ & Time \\
\hline 2 & 0.0232 & 0.000 & 0.0340 & 0.000 & 0.0428 & 0.000 & 0.0442 & 0.015 & 0.0385 & 0.015 \\
\hline 3 & 0.0027 & 0.000 & 0.0073 & 0.063 & 0.0172 & 0.063 & 0.0242 & 0.266 & 0.0360 & 0.250 \\
\hline 4 & 0.0002 & 0.078 & 0.0009 & 0.078 & 0.0040 & 0.328 & 0.0078 & 1.312 & 0.0209 & 10.532 \\
\hline 5 & 0.0000 & 1.484 & 0.0001 & 11.953 & 0.0007 & 11.953 & 0.0019 & 12.156 & 0.0090 & 339.860 \\
\hline 6 & 0.0000 & 1.844 & 0.0000 & 14.985 & 0.0001 & 14.844 & 0.0004 & 480.359 & 0.0034 & 61324.600 \\
\hline 7 & 0.0000 & 17.437 & 0.0000 & 557.141 & 0.0000 & 555.422 & 0.0000 & 555.921 & 0.0004 & 71302.900 \\
\hline
\end{tabular}

As suggested by the referee, we next compare (2.5) with Equation (11) of Furman (2007) by numerically calculating the computational time (in seconds) and $\mathrm{P}\left(S_{n}=x\right)$. The values of $\mathrm{P}\left(S_{n}=x\right)$ are calculated, using MATHEMATICA ${ }^{\circledR}$ 5.1, for some selected values of $\alpha_{j}=j$, $p_{j}=j / 10, x=3,5,8,10,15$, and $n=2, \ldots, 7$, and are given in Tables 1 and 2. Since Furman's formula (11) involves recurrence relations, the order of accuracy of the values in Table 2 is restricted to $10^{-3}$ to bring down the computational time. A comparison of the values in Tables 1 and 2 shows that the computation of probability values using (2.5) requires much less time than that of Furman's formula (11).

\subsection{Random parameter representation}

In this subsection we obtain a random parameter representation for the convolution of independent $\mathrm{CNB}$ variables and also of certain dependent $\mathrm{CP}$ variables.

Let $Y_{1}, Y_{2}, \ldots, Y_{n}$ be independent variables, where $Y_{j} \sim \operatorname{CNB}\left(\alpha_{j}, p_{j}, Q\right)$ for $1 \leq j \leq n$. We now introduce the following notation. Let

$$
\begin{gathered}
p_{m}=\max _{1 \leq j \leq n} p_{j}, \quad q_{m}=1-p_{m}, \quad s_{j}=\frac{q_{j}}{p_{j}}, \quad s_{l}=\min _{1 \leq j \leq n} s_{j}=\frac{q_{m}}{p_{m}} \\
\alpha=\sum_{j=1}^{n} \alpha_{j}, \quad c_{n}=\prod_{j=1}^{n}\left(\frac{s_{l}}{s_{j}}\right)^{\alpha_{j}}, \quad \text { and } \quad a_{i}=\frac{1}{i} \sum_{j=1}^{n} \alpha_{j}\left(1-\frac{s_{l}}{s_{j}}\right)^{i} \text { for } i \in \mathbb{Z}_{+} \backslash\{0\} .
\end{gathered}
$$

Define $K_{n}$ to be a $\mathbb{Z}_{+}$-valued random variable with probability distribution

$$
\mathrm{P}\left(K_{n}=k\right)=c_{n} b_{k} \quad \text { for } k \in \mathbb{Z}_{+},
$$

where $b_{0}=1$ and $b_{k}=(1 / k) \sum_{i=1}^{k} i a_{i} b_{k-i}$ for $k \geq 1$ (see Remark 2.2, below). 
We are now ready to prove the main result of this subsection. Note that our approach is essentially that of Furman (2007), except that he used the MGF, while we use the distribution itself.

Theorem 2.2. Let $Y_{j} \sim \operatorname{CNB}\left(\alpha_{j}, p_{j}, Q\right)$ for $1 \leq j \leq n$, and let $S_{n}=\sum_{j=1}^{n} Y_{j}$. Then $S_{n} \sim \mathrm{CNB}\left(K_{n}+\alpha, p_{m}, Q\right)$, where the distribution of $K_{n}$ is defined in (2.6).

Proof. Using (2.3),

$$
\begin{aligned}
\mathcal{L}\left(Y_{j}\right) & =\left(\delta_{0}-s_{j}\left(Q-\delta_{0}\right)\right)^{-\alpha_{j}} \\
& =\left(\left(\delta_{0}-s_{l}\left(Q-\delta_{0}\right)\right) \frac{s_{j}}{s_{l}}\left(\delta_{0}-\left(1-\frac{s_{l}}{s_{j}}\right)\left(\delta_{0}-s_{l}\left(Q-\delta_{0}\right)\right)^{-1}\right)\right)^{-\alpha_{j}} .
\end{aligned}
$$

Observe that $\delta_{0}-s_{l}\left(Q-\delta_{0}\right)$ is a finite signed measure and that $\left(\delta_{0}-s_{l}\left(Q-\delta_{0}\right)\right)^{-1}=$ $\mathrm{CG}\left(p_{m}, Q\right):=G$ (say). Therefore, from (2.7) we have

$$
\begin{aligned}
\mathcal{L}\left(S_{n}\right) & =\prod_{j=1}^{n} \mathcal{L}\left(Y_{j}\right) \\
& =\left(\delta_{0}-s_{l}\left(Q-\delta_{0}\right)\right)^{-\alpha} c_{n} \prod_{j=1}^{n}\left(\delta_{0}-\frac{1-s_{l} / s_{j}}{\delta_{0}-s_{l}\left(Q-\delta_{0}\right)}\right)^{-\alpha_{j}} \\
& =G^{\alpha} c_{n} \exp \left(\sum_{k=1}^{\infty} \frac{1}{k} \sum_{j=1}^{n} \alpha_{j}\left(1-\frac{s_{l}}{s_{j}}\right)^{k} G^{k}\right) \\
& =G^{\alpha} c_{n} \exp \left(\sum_{k=1}^{\infty} a_{k} G^{k}\right) \quad \text { (say). }
\end{aligned}
$$

If we write $f(z)=\exp \left(\sum_{k=1}^{\infty} a_{k} z^{k}\right)=\sum_{k=0}^{\infty} b_{k} z^{k}$ then $b_{k}=f^{(k)}(0) / k$ ! for $k \in \mathbb{Z}_{+}$, where $f^{(k)}$ denotes the $k$ th derivative of $f$. Therefore, it can be seen that $b_{0}=1, b_{1}=a_{1}$, and $b_{2}=a_{1}^{2} / 2+a_{2}$, and, in general, we obtain $b_{k}=(1 / k) \sum_{i=1}^{k} i a_{i} b_{k-i}$ for $k \geq 1$. Using these facts, we obtain, from (2.8),

$$
\begin{aligned}
\mathcal{L}\left(S_{n}\right) & =G^{\alpha} \sum_{k=0}^{\infty} c_{n} b_{k} G^{k} \\
& =G^{\alpha} \sum_{k=0}^{\infty} \mathrm{P}\left(K_{n}=k\right) G^{k} \\
& =\operatorname{CNB}\left(\alpha, p_{m}, Q\right) \operatorname{CNB}\left(K_{n}, p_{m}, Q\right) \\
& =\operatorname{CNB}\left(K_{n}+\alpha, p_{m}, Q\right),
\end{aligned}
$$

since $G^{\alpha}=\mathrm{CNB}\left(\alpha, p_{m}, Q\right)$. This proves the result.

Remark 2.2. Note that, from the proof of Theorem 2.2,

$$
f(z)=\exp \left(\sum_{k=1}^{\infty} a_{k} z^{k}\right)=\sum_{k=0}^{\infty} b_{k} z^{k} .
$$


Setting $z=1$ in the above equation, we obtain

$$
\begin{aligned}
\sum_{k=0}^{\infty} b_{k} & =\exp \left(\sum_{k=1}^{\infty} a_{k}\right) \\
& =\exp \left(\sum_{k=1}^{\infty} \frac{1}{k} \sum_{j=1}^{n} \alpha_{j}\left(1-\frac{s_{l}}{s_{j}}\right)^{k}\right) \\
& =\exp \left(-\sum_{j=1}^{n} \alpha_{j} \ln \left(\frac{s_{l}}{s_{j}}\right)\right) \\
& =\prod_{j=1}^{n}\left(\frac{s_{l}}{s_{j}}\right)^{-\alpha_{j}} \\
& =c_{n}^{-1} .
\end{aligned}
$$

Hence, $\mathrm{P}\left(K_{n}=k\right)=c_{n} b_{k}, k \in \mathbb{Z}_{+}$, is a valid probability distribution.

Corollary 2.1. (Furman (2007, Theorem 2).) Let $Y_{j} \sim \mathrm{NB}\left(\alpha_{j}, p_{j}\right)$ for $1 \leq j \leq n$, and let $S_{n}=\sum_{j=1}^{n} Y_{j}$. Then $S_{n} \sim \mathrm{NB}\left(K_{n}+\alpha, p_{m}\right)$.

Corollary 2.2. (Furman (2007, Theorem 1).) Let $Y_{j} \sim \mathrm{NB}\left(\alpha_{j}, p_{j}\right)$ for $1 \leq j \leq n$, and let $S_{n}=\sum_{j=1}^{n} Y_{j}$. Then

$$
\mathrm{P}\left(S_{n}=x\right)=\sum_{k=0}^{\infty} c_{n} b_{k}\left(\begin{array}{c}
\alpha+k+x-1 \\
x
\end{array}\right) p_{m}^{\alpha+k} q_{m}^{x}, \quad x \in \mathbb{Z}_{+} .
$$

Proof. The proof easily follows from Corollary 2.1 and the fact that

$$
\mathrm{P}\left(S_{n}=x\right)=\sum_{k=0}^{\infty} \mathrm{P}\left(S_{n}=x \mid K_{n}=k\right) \mathrm{P}\left(K_{n}=k\right) .
$$

Next we look at the case of different $Q_{j}$ s. In this case, a CP representation is useful.

Theorem 2.3. Let $W_{1}, W_{2}, \ldots, W_{n}$ be independent $\operatorname{CNB}\left(\alpha_{j}, p_{j}, Q_{j}\right)$ random variables, and let $T_{n}=\sum_{j=1}^{n} W_{j}$. Also, let $\lambda_{j}=-\alpha_{j} \ln p_{j}, q_{j}=\left(1-p_{j}\right)$, and $G_{j}=\ln \left(\delta_{0}-q_{j} Q_{j}\right) / \ln p_{j}$. Then $T_{n} \sim \mathrm{CP}(\lambda, G)$, where $\lambda=\sum_{j=1}^{n} \lambda_{j}$ and $G=(1 / \lambda) \sum_{j=1}^{n} \lambda_{j} G_{j}$.

Proof. Note that

$$
\begin{aligned}
\mathcal{L}\left(W_{j}\right) & =\left(\delta_{0}-\frac{q_{j}}{p_{j}}\left(Q_{j}-\delta_{0}\right)\right)^{-\alpha_{j}} \\
& =\left(\frac{p_{j}}{\delta_{0}-q_{j} Q_{j}}\right)^{\alpha_{j}} \\
& =\exp \left(\alpha_{j}\left(\ln \left(1-q_{j}\right)-\ln \left(\delta_{0}-q_{j} Q_{j}\right)\right)\right) \\
& =\exp \left(-\alpha_{j} \ln \left(1-q_{j}\right)\left(\frac{\ln \left(\delta_{0}-q_{j} Q_{j}\right)}{\ln \left(1-q_{j}\right)}-\delta_{0}\right)\right) \\
& =\operatorname{CP}\left(\lambda_{j}, G_{j}\right),
\end{aligned}
$$


where $\lambda_{j}=-\alpha_{j} \ln p_{j}$ and $G_{j}=\ln \left(\delta_{0}-q_{j} Q_{j}\right) / \ln p_{j}$. Note that if $W_{j} \sim \operatorname{CP}\left(\lambda_{j}, G_{j}\right)$ then, by the additivity property, $T_{n}=\sum_{j=1}^{n} W_{j} \sim \mathrm{CP}(\lambda, G)$, where $\lambda=\sum_{j=1}^{n} \lambda_{j}$ and $G=(1 / \lambda) \sum_{j=1}^{n} \lambda_{j} G_{j}$. The result now follows.

Remarks 2.3. Here we discuss the connections between CP and CNB distributions.

(i) Suppose that $Y \sim \operatorname{CP}(\lambda, F)$ so that $\mathcal{L}(Y)=\exp \left(\lambda\left(F-\delta_{0}\right)\right)$. Equating this distribution to a $\operatorname{CNB}(\alpha, p, Q)$ distribution and then solving for $p$ and $Q$, using (2.10), we obtain

$$
p=\mathrm{e}^{-\lambda / \alpha} \quad \text { and } \quad Q=\frac{\delta_{0}-\exp (-(\lambda / \alpha) F)}{1-\mathrm{e}^{-\lambda / \alpha}},
$$

where $\alpha>0$ is arbitrary and $Q$ is in general a finite signed measure.

(ii) Applying Theorem 2.3 and then using part (i), we obtain $\mathcal{L}\left(T_{n}\right)=\mathrm{CNB}(\alpha, p, Q)$, where

$$
p=\prod_{j=1}^{n} p_{j}^{\alpha_{j} / \alpha}, \quad Q=\frac{\delta_{0}-\prod_{j=1}^{n}\left(\delta_{0}-q_{j} Q_{j}\right)^{-\alpha_{j} / \alpha}}{1-\prod_{j=1}^{n} p_{j}^{\alpha_{j} / \alpha}}
$$

and $\alpha>0$ is arbitrary. Since $Q$ is in general a finite signed measure, a CP representation given in Theorem 2.3 may be useful for applications.

(iii) Let $N$ follow a logarithmic series distribution with parameter $0<q<1$ so that

$$
\mathrm{P}(N=k)=\frac{q^{k}}{k h(q)}, \quad k=1,2, \ldots,
$$

where $h(q)=-\ln (1-q)$. Also, let the $X_{i}$ be i.i.d. with distribution $Q$. Then the distribution of $\sum_{i=1}^{N} X_{i}$ is a compound logarithmic series distribution, denoted by $\operatorname{CL}(q, Q)$. That is,

$$
\mathrm{CL}(q, Q)=\frac{\ln \left(\delta_{0}-q Q\right)}{\ln (1-q)} .
$$

When $F=\operatorname{CL}(q, Q)$, we have $\mathrm{CP}(\lambda, F)=\mathrm{CNB}(-\lambda / \ln (1-q), 1-q, Q)$ and $Q$ is now a probability measure.

\subsection{Sums of dependent $C P$ variables}

We consider here the sums of certain dependent CP distributions, where the dependence is caused by a common mixing random variable $W$. Such a case arises in the distribution of combined portfolios. For example, Dhaene et al. (2003) considered the case of $W$ being a gamma variable.

In the sequel, $X \stackrel{\mathscr{L}}{=} Y$ means that the distributions of $X$ and $Y$ are the same.

Theorem 2.4. Let $W>0$ be a continuous random variable, let $\left\{N_{i}(t)\right\}, 1 \leq i \leq n$, be independent Poisson processes with rate $\lambda_{i}$, and let $V_{i}:=N_{i}(W), 1 \leq i \leq n$. Define $S_{V_{i}}:=\sum_{j=1}^{V_{i}} X_{i, j}$, where $X_{i, j} \sim Q_{i}$. Then $U_{n}=\sum_{i=1}^{n} S_{V_{i}} \stackrel{\mathcal{L}}{=} \sum_{i=1}^{N(W)} X_{j} \sim \operatorname{CP}(\lambda W, Q)$, where $\{N(t)\}$ is a Poisson process with parameter $\lambda=\sum_{i=1}^{n} \lambda_{i}$ and $X_{j} \sim Q=(1 / \lambda) \sum_{i=1}^{n} \lambda_{i} Q_{i}$.

Proof. Observe that, for any given $W>0, S_{V_{i}} \sim \operatorname{CP}\left(\lambda_{i} W, Q_{i}\right)$ for $1 \leq i \leq n$. By the additivity property of $\mathrm{CP}$ distributions, we obtain $\left(U_{n} \mid W\right) \sim \operatorname{CP}(\lambda W, Q)$, and, hence, (unconditionally also) $U_{n} \sim \mathrm{CP}(\lambda W, Q)$, where $\lambda=\sum_{i=1}^{n} \lambda_{i}$ and $Q=(1 / \lambda) \sum_{i=1}^{n} \lambda_{i} Q_{i}$. 


\section{Convolution of weighted gamma random variables}

The distribution of the sum of weighted gamma random variables arises in many situations, and does not admit a closed form (see Diaconis and Perlman (1990)). As an application of Theorem 2.2, here we obtain the random parameter representation for such sums. This representation is compact and may be helpful for analytical purposes.

Theorem 3.1. Let $Z_{1}, Z_{2}, \ldots, Z_{n}$ be independent random variables, where $Z_{j} \sim G\left(\beta_{j}, t_{j}\right)$, the gamma distribution with scale parameter $\beta_{j}^{-1}$ and shape parameter $t_{j}>0$. For $c_{j}>0$ and $i \in \mathbb{Z}_{+} \backslash\{0\}$, let $T_{n}=\sum_{j=1}^{n} c_{j} Z_{j}, \beta=\max _{1 \leq j \leq n} \beta_{j} /\left(c_{j}+\beta_{j}\right), d_{n}=\prod_{j=1}^{n}((1-$ $\left.\beta) \beta_{j} /\left(c_{j} \beta\right)\right)^{t_{j}}$, and $a_{i}=(1 / i) \sum_{j=1}^{n} t_{j}\left(1-(1-\beta) \beta_{j} /\left(c_{j} \beta\right)\right)^{i}$. Then $T_{n} \sim G(\beta /(1-\beta)$, $\left.L_{n}+t\right)$, where $L_{n}$ is a random variable with $\mathrm{P}\left(L_{n}=k\right)=d_{n} b_{k}, k \in \mathbb{Z}_{+}$, and $t=\sum_{j=1}^{n} t_{j}$. Here, $b_{0}=1$ and $b_{k}=(1 / k) \sum_{i=1}^{k} i a_{i} b_{k-i}$ for $k \in \mathbb{Z}_{+} \backslash\{0\}$.

Proof. Note that

$$
Z_{j} \sim G\left(\beta_{j}, t_{j}\right) \Longleftrightarrow c_{j} Z_{j} \sim G\left(\frac{\beta_{j}}{c_{j}, t_{j}}\right) \Longleftrightarrow N\left(c_{j} Z_{j}\right) \sim \mathrm{NB}\left(t_{j}, \frac{\beta_{j}}{c_{j}+\beta_{j}}\right)
$$

(see Proposition 2 of Engel and Zijlstra (1980)), where $\{N(t)\}$ is a standard (parameter unity) Poisson process. Also, there exist (see Vellaisamy and Sreehari (2008)) independent standard Poisson processes $\left\{N_{j}(t)\right\}_{1 \leq j \leq n}$ and $\{N(t)\}$ such that

$$
N_{1}\left(c_{1} Z_{1}\right)+N_{2}\left(c_{2} Z_{2}\right)+\cdots+N_{n}\left(c_{n} Z_{n}\right) \stackrel{\mathscr{L}}{=} N\left(c_{1} Z_{1}+c_{2} Z_{2}+\cdots+c_{n} Z_{n}\right) .
$$

By Corollary 2.1 we have $N\left(c_{1} Z_{1}+c_{2} Z_{2}+\cdots+c_{n} Z_{n}\right)=N\left(T_{n}\right) \sim \mathrm{NB}\left(L_{n}+t, \beta\right)$, where $t$ and $\beta$ are as defined in the theorem and $L_{n}$ is the discrete random variable with $\mathrm{P}\left(L_{n}=k\right)=d_{n} b_{k}$ for $k \in \mathbb{Z}_{+}$. Hence, $T_{n} \sim G\left(\beta /(1-\beta), L_{n}+t\right)$, which proves the result.

Remark 3.1. When $c_{1}=c_{2}=\cdots=c_{n}=1$, Theorem 3.1 yields the convolution of $n$ independent gamma variables with arbitrary parameters. It is known in the literature (see, for example, Sim (1992, p. 140)) that the density of $T_{n}$ is complicated. Our Theorem 3.1 gives a simple random parameter representation for the distribution of $T_{n}$, which may be helpful for analytical or inferential purposes.

\section{Examples and applications}

In this section we discuss some examples and applications of the results derived in Sections 2 and 3. We start with an application of Theorem 2.2 to risk theory. The finite sums of CNB random variables naturally occur in credit risk modeling and have been studied by many authors (see, for example, Gundlach and Lehrbass (2004, pp. 32-40) and Dhaene et al. (2003)).

Example 4.1. (Total claim amount.) Let the claim sizes $X_{i} \sim E(\beta)=Q$, the exponential distribution with parameter $\beta$. Suppose that a company has a portfolio of $n$ policies, and assume that the number $N_{i}$ of claims of the $i$ th policy follows $\mathrm{NB}\left(\alpha_{i}, p_{i}\right)$, which is a reasonable model, especially when $\operatorname{var}\left(N_{i}\right)>\mathrm{E}\left(N_{i}\right)$. Our interest is in the distribution of the total claim amount defined by

$$
S_{n}=\sum_{i=1}^{n} \sum_{j=1}^{N_{i}} X_{j}
$$


An application of Theorem 2.2 shows that

$$
\begin{aligned}
\mathcal{L}\left(S_{n}\right) & =\operatorname{CNB}\left(K_{n}+\alpha, p_{m}, Q\right) \\
& =\sum_{k=0}^{\infty} \mathrm{CNB}\left(k+\alpha, p_{m}, Q\right) \mathrm{P}\left(K_{n}=k\right) .
\end{aligned}
$$

Also, the density of $S_{n}$ is

$$
f_{S_{n}}(x)=\sum_{k=0}^{\infty} c_{n} b_{k} \sum_{l=0}^{\infty}\left(\begin{array}{c}
\alpha+k+l-1 \\
l
\end{array}\right) p_{m}^{\alpha+k} q_{m}^{l} f_{T_{l}}(x),
$$

where

$$
f_{T_{l}}(x)=\frac{\beta^{l}}{\Gamma(l)} \mathrm{e}^{-\beta x} x^{l-1} \quad \text { for } x>0
$$

is the density of the gamma $G(\beta, l)$ variable, since $Q^{l}=G(\beta, l)$.

Panjer and Wilmot (1981) considered the case in which $n=1$ and suggested an approximation procedure employing the methods of numerical analysis to evaluate the error in approximating $S_{1}$ to a compound binomial distribution. Our expression (4.1) gives the exact density of $S_{n}$.

The following two examples correspond to Theorem 2.4.

Example 4.2. Let $W \sim G(\beta, s)$ in Theorem 2.4 so that $V_{i}=N_{i}(W) \sim \mathrm{NB}\left(s, \beta /\left(\beta+\lambda_{i}\right)\right)$ and $S_{V_{i}} \sim \mathrm{CNB}\left(s, \beta /\left(\beta+\lambda_{i}\right), Q_{i}\right)$. Then, by Theorem $2.4, U_{n}=\sum_{i=1}^{n} S_{V_{i}} \sim \operatorname{CNB}(s, \beta /(\beta+$ $\lambda$ ), $Q$ ), where $Q$ and $\lambda$ are as defined in Theorem 2.4. This result is due to Dhaene et al. (2003).

Example 4.3. Let $W \sim \mathrm{L}(\alpha)$, the Lindley distribution with parameter $\alpha$ (see Johnson et al. (2005)), with density

$$
f_{W}(x)=\frac{\alpha^{2}}{\alpha+1}(1+x) \mathrm{e}^{-\alpha x}, \quad x>0, \alpha>0 .
$$

It is well known that $N_{i}(W) \sim \operatorname{PL}\left(\alpha, \lambda_{i}\right)$, the Poisson-Lindley distribution with parameters $\alpha$ and $\lambda_{i}$ having distribution

$$
\mathrm{P}\left(N_{i}(W)=k\right)=\frac{\alpha^{2}}{\alpha+1} \frac{\lambda_{i}^{k}\left(\alpha+\lambda_{i}+k+1\right)}{\left(\alpha+\lambda_{i}\right)^{k+2}}, \quad k \in \mathbb{Z}_{+} .
$$

Let $U_{n}, \lambda$, and $Q$ be defined as in Theorem 2.4. Then $U_{n}$ follows compound $\operatorname{PL}(\alpha, \lambda, Q)$.

Finally, we discuss two important applications of Theorem 3.1.

\subsection{Reliability of the $\boldsymbol{m}$-out-of- $\boldsymbol{n}$ :G system with different failure rates}

Consider an $m$-out-of- $n$ :G system with $n$ i.i.d. components having exponential $E(\lambda)$ lifetimes. Initially, each component has failure rate $\lambda_{0}$. As the first component fails, there is an increase in the stress on the remaining $(n-1)$ components, which increases the failure rate of the components to $\lambda_{1}$. Generally, the failure of the $i$ th component raises the stress on the remaining $(n-i)$ components, which increases the failure rate to $\lambda_{i}$. Our interest is to find the distribution of the system time to failure. 
Let $T_{i}$ denote the time to failure of the $i$ th component, and let $X_{i}=T_{i}-T_{i-1}$. Then, the time to system failure $T=T_{n-m+1}=\sum_{i=1}^{n-m+1} X_{i}$. Observe that $X_{i} \sim E\left(\alpha_{i}\right)$, where $\alpha_{i}=(n-i+1) \lambda_{i-1}$, and the reliability of the system is $R(t)=\mathrm{P}(T>t)$. Using Theorem 3.1, $T \sim G\left(\gamma, L_{n-m+1}+(n-m+1)\right)$, where $\gamma=\max _{i}(n-i+1) \lambda_{i-1}$.

Let $Z_{j} \sim G\left(\beta_{j}, t_{j}\right)$. Indeed, Scheuer (1988) derived the distribution of $T_{n}=\sum_{i=1}^{n} Z_{j}$ as

$$
f_{T_{n}}(x)=B \sum_{k=1}^{n} \sum_{m=1}^{t_{k}} \frac{\Phi_{k m}\left(-\beta_{j}\right)}{\left(t_{k}-m\right) !(m-1) !} x^{t_{k}-1} \exp \left(-\beta_{j} x\right),
$$

where $B=\prod_{j=1}^{n} \beta_{j}^{t_{j}}$ and

$$
\Phi_{k m}(x)=\frac{\mathrm{d}^{m-1}}{\mathrm{~d} x^{m-1}} \prod_{\substack{j=1 \\ j \neq k}}^{n}\left(\beta_{j}+x\right)^{-t_{j}} .
$$

Using Theorem 3.1, we see that $T_{n} \sim G\left(\gamma, L_{n}+t\right)$ with

$$
\begin{aligned}
f_{T_{n}}(x) & =\sum_{k=0}^{\infty} \mathrm{P}\left(L_{n}=k\right) \frac{\gamma^{t+k}}{\Gamma(t+k)} \mathrm{e}^{-\gamma x} x^{t+k-1} \\
& =\sum_{k=0}^{\infty} d_{n} b_{k} \frac{\gamma^{t+k}}{\Gamma(t+k)} \mathrm{e}^{-\gamma x} x^{t+k-1},
\end{aligned}
$$

where $\gamma=\beta /(1-\beta), \beta=\max _{1 \leq j \leq n} \beta_{j} /\left(1+\beta_{j}\right)$, and $d_{n}$ and the $b_{k}$ s are defined in Theorem 3.1. Note that (4.3) involves derivatives of the $m$ th order and, hence, (4.2) and (4.3) are difficult to compute. Equation (4.4) is much simpler and can be easily evaluated.

\subsection{The shortest path problem in graph theory}

The shortest path from a source node to a destination node is a path which minimizes the sum of the positive weights of its constituent links. The related shortest path tree (SPT) is the union of the shortest paths from the source node to a set of $m$ other nodes in the graph of $r$ nodes. If $m=r-1$, the SPT connects all nodes and is called a spanning tree. The uniform recursive tree (URT) of size $r$ is a random tree rooted at node $A$ and, at each stage, a new node is attached uniformly to the existing node until all the nodes are discovered. We analyze the influence of the link weight structure on the SPT. Such problems arise in communication networks (see, for example, Mieghem (2006, pp. 347-384)).

The problem of finding the shortest path between two nodes $A$ and $B$ in a complete graph $K_{r}$, with link weights as $E(1)$ (exponentially distributed with mean 1) variables, can be modeled in the form of a Markov discovery process $\{X(t)\}$ with state space $S=\{1,2, \ldots, r\}$, where $X(t)$ denotes the number of nodes discovered up to time $t$. Note that $X\left(t_{0}\right)=A, X(T)=B$, where $t_{0}$ is the starting time and $T$ denotes the random time to reach $B$, and that the transmission rates are $\lambda_{j}=j(r-j), j \in S$. This is because, from the first node $A, r-1$ new nodes can be reached, each with $E(1)$ link weights, and so the shortest path $Z_{1} \sim E(r-1)$. Similarly, from the first two nodes, the remaining $r-2$ nodes can be reached with the shortest path $Z_{2} \sim E(2(r-2))$. In general, from the $(j-1)$ th node to the $j$ th node, the shortest path $Z_{j} \sim E\left(\lambda_{j}\right)$ with $\lambda_{j}=j(r-j)$.

Observe that the time to reach the $k$ th node from the source node $A$ or the discovery time of the $k$ th node is given by $M_{k}=\sum_{j=1}^{k} Z_{j}$. Using the MGF of $M_{k}$, the mean $\mathrm{E}\left(M_{k}\right)$ and the variance $\operatorname{var}\left(M_{k}\right)$ are computed (see Mieghem (2006, p. 359)), but they are rather complicated. 
An application of Theorem 3.1 yields $M_{k} \sim G\left(\beta /(1-\beta), L_{k}+k\right)$, where

$$
\beta=\max _{1 \leq j \leq n} \frac{j(r-j)}{1+j(r+j)} .
$$

Note that $\beta /(1-\beta)=k(r-k)$ if $k \leq r / 2$. When $k \geq r / 2$, we have $\beta /(1-\beta)=r^{2} / 4$ if $r$ is even and $\beta /(1-\beta)=(r-1)^{2} / 4$ if $r$ is odd. Therefore, the density of $M_{k}$ is

$$
f_{M_{k}}(x)=\sum_{m=0}^{\infty} d_{k} b_{m} \frac{\gamma^{k+m}}{\Gamma(k+m)} \mathrm{e}^{-\gamma x} x^{k+m-1},
$$

where $\gamma=\beta /(1-\beta)$, as described above.

It is well known (see Mieghem (2006, p. 359)) that the shortest path in a complete graph with exponential $E(1)$ link weights is a URT. Now, let $W_{r}$ denote the length of the shortest path in $K_{r}$, and let $N$ denote the number of nodes, excluding the source node, discovered by the URT to reach the destination node. Then

$$
W_{r}=\sum_{j=1}^{N} Z_{j}
$$

where $N$ follows a discrete uniform distribution over $\{1,2, \ldots, r-1\}$ and is independent of the $Z_{j}$. Also, the density of $W_{r}$ is given by

$$
f_{W_{r}}(x)=\sum_{k=1}^{r-1} \mathrm{P}(N=k) f_{M_{k}}(x)=\frac{1}{r-1} \sum_{k=1}^{r-1} f_{M_{k}}(x),
$$

where $f_{M_{k}}(x)$ is defined in (4.5). It is mentioned in Mieghem (2006, p. 360) that the density of $W_{r}$ can be obtained by using the inverse Laplace transform of the MGF of $W_{r}$. Equation (4.7) gives the exact density of $W_{r}$. Note that the moments and other characteristics of $W_{r}$ can easily be computed using (4.6) or (4.7).

\section{Concluding remarks}

The main contributions of this paper are the derivation of an exact expression and the random parameter representation for the convolution of compound negative binomial variables. In the case of negative binomial distributions, it is numerically verified that the exact expression is computationally more efficient than the random parametric form. Some applications to insurance mathematics are also discussed. The distribution of a sum of certain dependent compound Poisson variables is obtained, which generalizes some existing results. The conditions under which a compound Poisson distribution is also a compound negative binomial distribution are analyzed. Using the connection between negative binomial and gamma distributions, the convolution of arbitrary gamma variables is derived, which is also a useful result. This result is then applied to two important practical problems which arise in reliability theory and graph theory.

\section{Acknowledgements}

The authors are grateful to the anonymous referee for his/her encouraging remarks and several suggestions which improved the presentation of the results. The second author thanks CSIR for support through research grant 09/087(051)/2006-EMR-I. 


\section{References}

Dhaene, J. et al. (2003). On the computation of the capital multiplier in the Fortis credit economic capital model. Belg. Actuarial Bull. 3, 50-57.

Diaconis, P. AND PERLMAn, M. D. (1990). Bounds for tail probabilities of weighted sums of independent gamma random variables. In Topics In Statistical Dependence (IMS Lecture Notes Monogr. Ser. 16), Institute of Mathematical Statistics, Hayward, CA, pp. 147-166.

Drekic, S. AND Willmot, G. E. (2005). On the moments of the time of ruin with applications to phase-type claims. N. Amer. Actuarial J. 9, 17-30.

ENGEL, J. ANd ZiJlstra, M. (1980). A characterization of the gamma distribution by the negative binomial distribution. J. Appl. Prob. 17, 1138-1144.

Furman, E. (2007). On the convolution of negative binomial random variables. Statist. Prob. Lett. 77, $169-172$.

Gundlach, M. and Lehrbass, F. (2004). CreditRisk ${ }^{+}$in the Banking Industry. Springer, New York.

Johnson, N. L., Kemp, A. W. And Kotz, S. (2005). Univariate Discrete Distributions, 3rd edn. John Wiley, Hoboken, NJ.

Mieghen, P. V. (2006). Performance Analysis of Communication Networks and Systems. Cambridge University Press.

Panjer, H. H. and Willmot, G. E. (1981). Finite sum evaluation of negative binomial-exponential model. ASTIN Bull. 12, 133-137.

Scheuer, E. M. (1988). Reliability of an $m$-out-of- $n$ system when component failure induces higher failure rates in survivors. IEEE Trans. Reliab. 37, 73-74.

Sim, C. H. (1992). Point processes with correlated gamma interarrival times. Statist. Prob. Lett. 15, $135-141$.

Vellaisamy, P. and Sreehari, M. (2008). Some intrinsic properties of the gamma distribution. Submitted.

Vellaisamy, P. and Upadhye, N. S. (2007). On the negative binomial distributions and its generalizations. Statist. Prob. Lett. 77, 173-180. 